\title{
Alterações cognitivas na SAOS
}

\author{
Cognitive impairment in obstructive sleep apnea syndrome
}

Pedro Felipe Carvalhedo de Bruin, Mauricio da Cunha Bagnato

\begin{abstract}
Resumo
Alterações da cognição e do desempenho estão bem estabelecidas em pacientes com SAOS, causando um impacto significativo sobre a qualidade de vida e o risco de acidentes nesses indivíduos. Tais alterações são mais profundas nos quadros mais graves de SAOS, o que explica a aparente discrepância na frequência e gravidade desse prejuízo entre estudos com pacientes de clínicas de sono e estudos de base populacional. Vários aspectos podem estar comprometidos, incluindo o processamento cognitivo, a atenção sustentada, as funções executivas e a memória. Entretanto, os mecanismos causais desses déficits não estão inteiramente elucidados, e existem controvérsias, particularmente em relação à contribuição relativa da hipóxia intermitente e da interrupção do sono presentes na SAOS. 0 impacto da sonolência diurna sobre o desempenho desses pacientes nos diversos testes cognitivos também ainda deve ser determinado, assim como o possível efeito de comorbidades frequentes, incluindo o diabete melito, a hipertensão arterial sistêmica, a doença cardiovascular e a obesidade. Existem evidências convincentes de que o tratamento com CPAP produz uma significativa melhora do desempenho e da cognição, sobretudo nos portadores de SAOS moderada e grave, embora sejam necessários mais estudos acerca do seu impacto a longo prazo.
\end{abstract}

Descritores: Apneia do sono tipo obstrutiva; Cognição; Função executiva; Memória; Pressão positiva contínua nas vias aéreas.

\begin{abstract}
Cognitive and performance impairment is well established in patients with obstructive sleep apnea syndrome (OSAS), having a significant impact on the quality of life and the risk of accidents in these individuals. The severity of the impairment correlates with that of the OSAS, which explains the apparent discrepancy between studies using patients from sleep clinics and population-based studies in terms of the reported frequency and severity of such impairment. Cognitive processing, sustained attention, executive functioning, and memory have all been reported to be impaired in OSAS. However, the causal mechanisms of these deficits have not been entirely clarified, and the relative contribution of intermittent hypoxia and sleep disruption in OSAS is particularly controversial. The potential effect of daytime sleepiness on the performance of these patients on various cognitive tests has yet to be determined, as does that of common comorbidities, such as diabetes, systemic arterial hypertension, cardiovascular disease, and obesity. There is compelling evidence that CPAP treatment can improve performance and cognition, particularly in mild to moderate cases, although further studies on the long-term impact of this type of treatment are still needed.
\end{abstract}

Keywords: Sleep apnea, obstructive; Cognition; Executive function; Memory; Continuous positive airway pressure.

\section{Introdução}

As alterações cognitivas na SAOS podem ser múltiplas e variadas, incluindo déficit do processamento cognitivo, de memória, de atenção e das funções executivas. ${ }^{(1,2)}$ Os acidentes de trânsito e de trabalho, importantes marcadores desse tipo de alteração, representam uma significativa causa de morbidade e mortalidade nesses pacientes.

Inquéritos de base populacional indicam que a SAOS reduz a qualidade de vida e que mesmo os casos classificados como leves apresentam dificuldades na realização de tarefas cotidianas. $^{(3-5)}$ Embora seja difícil estabelecer com segurança uma relação linear entre o grau de apneia e alterações neurocognitivas, a maioria dos estudos relata prejuízos mais evidentes nos casos mais graves. ${ }^{(5)}$

\section{Caracterização do déficit cognitivo na SAOS}

Para uma melhor compreensão das alterações cognitivas e comportamentais, torna-se útil que esses fenômenos sejam abordados a partir de uma perspectiva categórica. Resumidamente, os efeitos de SAOS podem incluir alterações do 
processamento cognitivo, da atenção sustentada e da atenção dividida, das funções executivas e, muito provavelmente, da memória (Quadro 1). ${ }^{(6)}$

\section{Processamento cognitivo}

Pacientes com SAOS apresentam um comprometimento do processamento cognitivo, ou seja, da habilidade de digerir a informação, o que ocasiona uma lentificação da execução de tarefas, aumento do número de erros e redução de respostas completas e do número de respostas por unidade de tempo, quando comparados a controles saudáveis pareados por idade e grau de instrução. ${ }^{(7)}$ Estudos de base populacional envolvendo um maior número de participantes, entretanto, têm evidenciado alterações menos marcantes que os estudos com pacientes recrutados em clínicas ou centros especializados em distúrbios do sono, provavelmente, refletindo um viés de recrutamento de indivíduos mais sintomáticos. ${ }^{(8)}$

\section{Memória}

Vários processos psicológicos estão envolvidos na memória, incluindo o registro, a memória de curto prazo, os processos de repetição, a memória de longo prazo e a recuperação da informação armazenada. Registro, ou memória sensorial, é o primeiro reconhecimento do estímulo e serve como evidência do estado de consciência. A redução do grau de alerta constitui uma evidência do comprometimento do registro na SAOS. Além desse aspecto da memória, outros, incluindo a capacidade de armazenar, reter e recuperar informações, podem estar comprometidos. Estudos de memória em pacientes com SAOS têm revelado resultados não muito consistentes, provavelmente em decorrência de diferenças no processo de amostragem ou na comparação a grupos controle normais ou a valores normativos. $^{(7,9)}$

\section{Atenção}

A atenção envolve diversos processos e capacidades que refletem o modo como os estímulos são recebidos e processados. A atenção pode ser sustentada (tônica), como é o caso na vigilância, ou fásica, variando com a mudança do estímulo. A atenção sustentada, expressão que algumas vezes é usada como sinônimo de concentração, é a capacidade mais usada para a avaliação da sonolência diurna, e

Quadro 1 - Alterações cognitivas na SAOS.

\begin{tabular}{|c|c|}
\hline Conceito & Déficit \\
\hline \multirow{4}{*}{$\begin{array}{l}\text { Processamento } \\
\text { cognitivo }\end{array}$} & Habilidade reduzida para digerir informação \\
\hline & - Lentificação na execução da tarefa \\
\hline & - Aumento dos erros \\
\hline & - Declínio nas respostas corretas e/ou completas por unidade de tempo \\
\hline Memória & Habilidade diminuída para registrar, armazenar, reter e recuperar informações \\
\hline \multirow{6}{*}{$\begin{array}{l}\text { Atenção sustentada } \\
\text { ou vigilância }\end{array}$} & Incapacidade de manter a atenção ao longo do tempo \\
\hline & - Prolongamento do tempo de resposta (tempo na tarefa) \\
\hline & - Aumento dos erros \\
\hline & - Queda dos melhores tempos de resposta \\
\hline & - Períodos de resposta lenta ou ausência de resposta (lapsos) \\
\hline & - Respostas na ausência do estímulo (respostas falsas) \\
\hline Atenção dividida & $\begin{array}{l}\text { Incapacidade de responder a mais de uma tarefa ou estímulo, tal como, ao conduzir um } \\
\text { veículo }\end{array}$ \\
\hline \multirow[t]{6}{*}{ Função executiva } & Problemas na manipulação e processamento da informação \\
\hline & Planejamento e execução inadequados \\
\hline & Desorganização: prejuízo do julgamento e tomada de decisões \\
\hline & Inflexibilidade: labilidade emocional \\
\hline & Impulsividade \\
\hline & Dificuldade de manter a motivação \\
\hline
\end{tabular}

Modificado de Weaver et al. ${ }^{(6)}$ 
seu comprometimento associa-se ao aumento na frequência de acidentes. À medida que uma tarefa se prolonga, a capacidade de permanecer atento torna-se mais exigida, o que pode afetar o desempenho. Os pacientes com SAOS exibem um desempenho inicial em testes de atenção comparável ao de controles normais em tarefas de curta duração. Entretanto, com o aumento da duração da tarefa, ocorre uma instabilidade no desempenho, ocasionando um prolongamento do tempo de resposta, lapsos e respostas sem estímulo prévio. ${ }^{(10)}$

Outro aspecto da atenção diz respeito ao seu limite de capacidade, ou seja, o envolvimento em uma tarefa que requer atenção controlada pode interferir no desempenho de outra tarefa com requisitos semelhantes. Denomina-se atenção dividida a essa capacidade de responder a mais de uma tarefa ou estímulo. Testes de atenção dividida em simuladores de direção mostram que pacientes com SAOS colidem mais, apresentam mais erros por incapacidade de permanecer na faixa de tráfego e de localizar obstáculos e sinais luminosos. ${ }^{(10)}$

\section{Funções executivas}

As funções executivas são responsáveis por comportamentos intencionais, independentes e relacionados ao próprio indivíduo, sendo dependentes do funcionamento do lobo frontal. Mesmo em circunstâncias onde há considerável perda da função cognitiva, se as funções executivas estiverem preservadas, o indivíduo poderá continuar a levar uma vida autônoma e produtiva. Por outro lado, a perda das funções executivas torna o indivíduo incapaz de cuidar de si próprio, trabalhar independentemente e manter um relacionamento social normal, ainda que a função cognitiva propriamente dita permaneça intacta. 0 paciente com prejuízo das funções executivas carece de motivação, é incapaz de iniciar uma atividade e tem dificuldade de planejar e executar tarefas que requeiram um comportamento voltado para a consecução de um objetivo. Pode ocorrer também labilidade emocional, irritabilidade ou impulsividade. ${ }^{(6)}$

0 desempenho nos testes de funções executivas é um dos mais afetados pela SAOS. (11) Esse prejuízo costuma ser evidenciado por problemas de fluência verbal, planejamento, pensamento sequencial e habilidade construcional. ${ }^{(12)}$ Nos estudos em que os pacientes são recrutados de clínicas e centros especializados de sono, a queda no desempenho costuma variar entre moderada e grave, em comparação a controles normais. ${ }^{(13)}$ Estudos de base populacional, porém, têm mostrado um efeito menos intenso. ${ }^{(14)}$

\section{Mecanismos}

Evidências de que pacientes com apneia obstrutiva do sono podem apresentar redução da atenção e do desempenho cognitivo são abundantes na literatura. ${ }^{(1,2)}$ Entretanto, os mecanismos exatos de tais alterações permanecem insuficientemente compreendidos. 0 estudo desses mecanismos de causa e efeito é dificultado, particularmente, pelo fato de que portadores de SAOS frequentemente apresentam comorbidades, tais como obesidade, diabete melito ou hipertensão arterial sistêmica, que atuam como potenciais fatores de confusão. ${ }^{(15)}$

De modo geral, acredita-se que a morbidade neurocognitiva na SAOS seja secundária a dois tipos de estímulos lesivos. 0 primeiro diz respeito à perturbação da arquitetura do sono associada a cada apneia e seu microdespertar subsequente, resultando em sonolência diurna excessiva. 0 segundo relaciona-se ao dano ao sistema neurocognitivo causado pelo estresse oxidativo no tecido cerebral consequente aos episódios de hipóxia intermitente. ${ }^{(11)}$

\section{Estudos em animais}

Alterações neuronais e cerebrais variadas têm sido descritas em estudos animais. Em relação ao hipocampo, estrutura que, dentre outras funções, está relacionada aos processos de memória, foi relatado que episódios isolados de hipóxia promovem excitotoxicidade induzida por glutamato ${ }^{(16)}$ e que a hipóxia intermitente modula a ativação colinérgica das proteínas $\mathrm{G}$, mas não sua ativação opioide. ${ }^{(17)}$ Foi descrita lesão hipocampal (estresse oxidativo, inflamação e morte neuronal) associada a expressão reduzida de enzimas antioxidantes, ${ }^{(18)}$ e foram encontradas evidências de que a deficiência de óxido nítrico induzida pela hipóxia intermitente diminui a atividade dos canais de potássio de alta condutância ativados pelo cálcio em neurônios hipocampais. ${ }^{(19)}$ Foi relatado também que a hipóxia intermitente afeta o córtex cerebral ${ }^{(20)}$ e que a produção de espécies reativas de 
oxigênio desempenha um papel central na lesão celular neuronal.(21) Além disso, foi observado que a hipóxia crônica recorrente induz a lesão irreversível e funcionalmente significativa em grupos neuronais ativos na vigília. ${ }^{(22)}$

A morbidade neurocognitiva na apneia do sono obstrutiva tem sido estudada em roedores submetidos a hipóxia intermitente e/ou a alteração do padrão de sono. Foi demonstrado que os eventos hipóxicos recorrentes prejudicam a memória de trabalho espacial e 0 aprendizado. ${ }^{(20,21,23)}$ Por outro lado, alterações da atenção comparáveis aos déficits cognitivos e das funções executivas descritos em pacientes com SAOS foram observados em ratos submetidos a um protocolo de interrupção do sono. ${ }^{(24)}$ Estudos dos efeitos da combinação dessas duas modalidades de estímulo nocivo, hipóxia intermitente e interrupção do sono revelaram uma interação complexa entre hipóxia e restrição/privação de sono, destacando a importância do emprego de modelos realistas que combinem ambos os tipos de estímulo. ${ }^{(25,26)}$

\section{Estudos clínicos}

Diversos estudos acerca da relação entre função cognitiva e comportamental, sono, variáveis respiratórias e hipoxemia têm produzido resultados controversos. Alguns achados sugerem uma relação entre hipoxemia e atenção, funções executivas, percepção, organização, velocidade motora, memória e fluência verbal, enquanto outros parecem apoiar uma relação causal entre a fragmentação do sono e funções executivas. (12,27) Recentemente, foi descrita uma associação entre a frequência de microdespertares e o déficit de ativação cerebral, sugerindo que a redução do grau de alerta e a lentificação do tempo de resposta estão mais fortemente associados à fragmentação do sono que à hipóxia. ${ }^{(28)} A$ relação entre $\mathrm{IAH}$ e déficit cognitivo tem-se mostrado fraca na maioria dos estudos. ${ }^{(8,12,13,27)}$

Um estudo comparativo das funções cognitivas entre pacientes com SAOS e pacientes com DPOC que também sofrem com frequência de hipoxemia noturna mostrou um comprometimento do processamento cognitivo e da memória em ambas as condições. Contudo, os pacientes com SAOS apresentaram maior disfunção nos testes de atenção sustentada, reconhecidamente mais afetado pela sonolência. ${ }^{(29)}$
$\mathrm{Na}$ tentativa de explicar a relação entre hipoxemia e disfunção cognitiva e neurocomportamental, foi proposto um modelo teórico que costuma ser designado por modelo pré-frontal. Segundo esse modelo, as alterações do sono, a hipercapnia e a hipóxia intermitente observadas nos pacientes com SAOS gerariam estresse ao nível celular e bioquímico, resultando em desequilíbrio homeostático e alteração da viabilidade neuronal e glial em determinadas regiões do cérebro, primariamente no córtex pré-frontal, o que estaria de acordo com algumas das evidências descritas em modelos animais. Essas alterações fisiopatológicas desestabilizariam o sistema executivo, ocasionando perturbação comportamental. 0 prejuízo causado pelos transtornos do sono produziria um desempenho ineficiente; porém, não uma incapacidade completa. Em tais circunstâncias, ao tentar desempenhar funções de memória ou de atenção dividida, o sistema já debilitado se veria forçado a recrutar outros sistemas, o que poderia explicar o aumento da ativação pré-frontal, documentado em estudos de ressonância magnética. ${ }^{(11)} 0$ recente relato de que, para atingir um nível de desempenho em testes de memória semelhante a controles normais, os pacientes com SAOS necessitam promover um recrutamento muito maior em várias regiões cerebrais reforça essa ideia de um mecanismo de compensação neural, que tende a reverter após o tratamento efetivo da apneia do sono. ${ }^{(30)}$

Uma das principais críticas a esse modelo tem sido a de que a disfunção executiva, observada em diversos estudos, poderia ser apenas um efeito secundário da redução do grau de alerta sobre as funções cognitivas superiores e não o resultado de uma lesão na região pré-frontal devido à hipóxia intermitente. É necessário reconhecer que, embora a hipoxemia noturna seja capaz de provocar dano neuronal em casos de SAOS grave não tratada, não existe ainda uma evidência científica sólida indicando que ela cause lesão cerebral irreversível no conjunto dos pacientes com SAOS como um grupo. ${ }^{(31)}$ No entanto, seja qual for o mecanismo, a detecção e o tratamento precoce da SAOS são importantes para prevenir ou interromper as consequências neurocognitivas, assim como outras consequências clínicas associadas a essa condição.

\section{Efeitos do tratamento}

$\begin{array}{cccc}\text { Estudos } & \text { randomizados } & \text { controlados } \\ \text { comparando } & 0 & \text { tratamento com CPAP ao }\end{array}$


tratamento com sham CPAP ou àquele com comprimidos de placebo, pelo período de 30 dias, demonstraram que o uso de CPAP associa-se à melhora da sonolência avaliada subjetiva e objetivamente. ${ }^{(32,33)}$ Estudos empregando apenas grupos controle não tratados ou tratados conservadoramente, sem sham CPAP, relataram melhora da sonolência objetiva após uma noite, ${ }^{(34)}$ duas semanas ${ }^{(34)}$ e três meses $^{(35)}$ de uso de CPAP. Finalmente, uma meta-análise confirmou a melhora efetiva da sonolência, tanto objetiva quanto subjetiva, pelo uso de CPAP. ${ }^{(36)}$ Estudos adequadamente controlados indicam que o desempenho em simuladores de direção, a atenção sustentada e a atenção dividida são os aspectos que mais se beneficiam com o tratamento. ${ }^{(37,38)}$ Além de melhorar a capacidade de dirigir, ${ }^{(39)}$ CPAP tem sido associado também à melhora da vigilância ${ }^{(40,41)}$ e da flexibilidade mental. ${ }^{(41)}$ Embora estudos de neuroimagem (Doppler transcraniano, potenciais relacionados a eventos, ressonância magnética por espectroscopia e ressonância magnética estrutural e funcional) tenham demonstrado mudanças no fluxo sanguíneo cerebral, metabolismo, morfologia e ativação cortical em resposta a desafios cognitivos em pacientes com SAOS, sob o ponto de vista da neuroimagem, ainda não há resultados conclusivos da recuperação dessas alterações após o tratamento com CPAP. ${ }^{(42)}$

\section{Referências}

1. Quan SF, Wright R, Baldwin CM, Kaemingk KL, Goodwin JL, Kuo TF, et al. Obstructive sleep apnea-hypopnea and neurocognitive functioning in the Sleep Heart Health Study. Sleep Med. 2006;7(6):498-507.

2. Lis S, Krieger S, Hennig D, Röder C, Kirsch P, Seeger $\mathrm{W}$, et al. Executive functions and cognitive subprocesses in patients with obstructive sleep apnoea. J Sleep Res. 2008;17(3):271-80.

3. Baldwin CM, Griffith KA, Nieto FJ, O'Connor GT, Walsleben JA, Redline S. The association of sleepdisordered breathing and sleep symptoms with quality of life in the Sleep Heart Health Study. Sleep. 2001;24(1):96-105.

4. Young T, Peppard PE, Gottlieb DJ. Epidemiology of obstructive sleep apnea: a population health perspective. Am J Respir Crit Care Med. 2002;165(9):1217-39.

5. Finn L, Young T, Palta M, Fryback DG. Sleepdisordered breathing and self-reported general health status in the Wisconsin Sleep Cohort Study. Sleep. 1998;21(7):701-6.

6. Weaver TE, George CFP. Cognition and performance in patients with obstructive sleep apnea. In: Kryger MH, Roth T, Dement WC, editors. Principles and Practice of
Sleep Medicine. Philadelphia: Saunders/Elsevier; 2010. p. 1023-33.

7. Rouleau 1, Décary A, Chicoine AJ, Montplaisir J. Procedural skill learning in obstructive sleep apnea syndrome. Sleep. 2002;25(4):401-11.

8. Boland LL, Shahar E, lber C, Knopman DS, Kuo TF, Nieto FJ, et al. Measures of cognitive function in persons with varying degrees of sleep-disordered breathing: the Sleep Heart Health Study. J Sleep Res. 2002;11(3):265-72.

9. Beebe DW, Groesz L, Wells C, Nichols A, McGee K. The neuropsychological effects of obstructive sleep apnea: a meta-analysis of norm-referenced and case-controlled data. Sleep. 2003;26(3):298-307.

10. Weaver TE. Outcome measurement in sleep medicine practice and research. Part 2: assessment of neurobehavioral performance and mood. Sleep Med Rev. 2001;5(3):223-36.

11. Beebe DW, Gozal D. Obstructive sleep apnea and the prefrontal cortex: towards a comprehensive model linking nocturnal upper airway obstruction to daytime cognitive and behavioral deficits. J Sleep Res. 2002;11(1):1-16.

12. Sateia MJ. Neuropsychological impairment and quality of life in obstructive sleep apnea. Clin Chest Med. 2003;24(2):249-59.

13. Engleman HM, Kingshott RN, Martin SE, Douglas NJ. Cognitive function in the sleep apnea/hypopnea syndrome (SAHS). Sleep. 2000;23 Suppl 4:S102-8.

14. Kim HC, Young T, Matthews CG, Weber SM, Woodward AR, Palta M. Sleep-disordered breathing and neuropsychological deficits. A population-based study. Am J Respir Crit Care Med. 1997;156(6):1813-9.

15. Farré R, Montserrat JM, Navajas D. Morbidity due to obstructive sleep apnea: insights from animal models. Curr Opin Pulm Med. 2008;14(6):530-6.

16. Fung SJ, Xi MC, Zhang JH, Sampogna S, Yamuy J, Morales FR, et al. Apnea promotes glutamate-induced excitotoxicity in hippocampal neurons. Brain Res. 2007;1179:42-50.

17. Hambrecht VS, Vlisides PE, Row BW, Gozal D, Baghdoyan HA, Lydic R. Hypoxia modulates cholinergic but not opioid activation of $\mathrm{G}$ proteins in rat hippocampus. Hippocampus. 2007;17(10):934-42.

18. Hung MW, Tipoe GL, Poon AM, Reiter RJ, Fung ML. Protective effect of melatonin against hippocampal injury of rats with intermittent hypoxia. J Pineal Res. 2008;44(2):214-21.

19. Tjong YW, Li M, Hung MW, Wang K, Fung ML. Nitric oxide deficit in chronic intermittent hypoxia impairs large conductance calcium-activated potassium channel activity in rat hippocampal neurons. Free Radic Biol Med. 2008;44(4):547-57.

20. Burckhardt IC, Gozal D, Dayyat E, Cheng Y, Li RC, Goldbart AD, et al. Green tea catechin polyphenols attenuate behavioral and oxidative responses to intermittent hypoxia. Am J Respir Crit Care Med. 2008;177(10):1135-41.

21. Shan X, Chi L, Ke Y, Luo C, Qian S, Gozal D, et al. Manganese superoxide dismutase protects mouse cortical neurons from chronic intermittent hypoxia-mediated oxidative damage. Neurobiol Dis. 2007;28(2):206-15.

22. Zhu Y, Fenik P, Zhan G, Mazza E, Kelz M, Aston-Jones $\mathrm{G}$, et al. Selective loss of catecholaminergic wake active neurons in a murine sleep apnea model. J Neurosci. 2007;27(37):10060-71. 
23. Row BW, Kheirandish L, Cheng Y, Rowell PP, Gozal D. Impaired spatial working memory and altered choline acetyltransferase (CHAT) immunoreactivity and nicotinic receptor binding in rats exposed to intermittent hypoxia during sleep. Behav Brain Res. 2007;177(2):308-14.

24. McCoy JG, Tartar JL, Bebis AC, Ward CP, McKenna JT, Baxter MG, et al. Experimental sleep fragmentation impairs attentional set-shifting in rats. Sleep. 2007;30(1):52-60.

25. Perry JC, D'Almeida V, Lima MM, Godoi FR, Vital MA, Oliveira MG, et al. Intermittent hypoxia and sleep restriction: motor, cognitive and neurochemical alterations in rats. Behav Brain Res. 2008;189(2):37380.

26. Perry JC, D'Almeida V, Antunes IB, Tufik S. Distinct behavioral and neurochemical alterations induced by intermittent hypoxia or paradoxical sleep deprivation in rats. Prog Neuropsychopharmacol Biol Psychiatry. 2008;32(1):87-94.

27. Smith IE, Shneerson JM. Is the SF 36 sensitive to sleep disruption? A study in subjects with sleep apnoea. J Sleep Res. 1995;4(3):183-8.

28. Ayalon L, Ancoli-lsrael S, Aka AA, McKenna BS, Drummond SP. Relationship between obstructive sleep apnea severity and brain activation during a sustained attention task. Sleep. 2009;32(3):373-81.

29. Roehrs T, Merrion M, Pedrosi B, Stepanski E, Zorick F, Roth T. Neuropsychological function in obstructive sleep apnea syndrome (OSAS) compared to chronic obstructive pulmonary disease (COPD). Sleep. 1995;18(5):382-8.

30. Castronovo V, Canessa N, Strambi LF, Aloia MS, Consonni $\mathrm{M}$, Marelli S, et al. Brain activation changes before and after PAP treatment in obstructive sleep apnea. Sleep. 2009;32(9):1161-72.

31. Verstraeten E. Neurocognitive effects of obstructive sleep apnea syndrome. Curr Neurol Neurosci Rep. 2007;7(2):161-6.

32. Engleman HM, Martin SE, Kingshott RN, Mackay TW, Deary 1J, Douglas NJ. Randomised placebo controlled trial of daytime function after continuous positive airway pressure (CPAP) therapy for the sleep apnoea/ hypopnoea syndrome. Thorax. 1998;53(5):341-5.
33. Jenkinson C, Davies RJ, Mullins R, Stradling JR. Comparison of therapeutic and subtherapeutic nasal continuous positive airway pressure for obstructive sleep apnoea: a randomised prospective parallel trial. Lancet. 1999;353(9170):2100-5.

34. Lamphere J, Roehrs T, Wittig R, Zorick F, Conway WA, Roth T. Recovery of alertness after CPAP in apnea. Chest. 1989;96(6):1364-7.

35. Engleman HM, Cheshire KE, Deary 1J, Douglas NJ. Daytime sleepiness, cognitive performance and mood after continuous positive airway pressure for the sleep apnoea/hypopnoea syndrome. Thorax. 1993;48(9):911-4.

36. Patel SR, White DP, Malhotra A, Stanchina ML, Ayas NT. Continuous positive airway pressure therapy for treating sleepiness in a diverse population with obstructive sleep apnea: results of a meta-analysis. Arch Intern Med. 2003;163(5):565-71.

37. George CF, Boudreau AC, Smiley A. Effects of nasal CPAP on simulated driving performance in patients with obstructive sleep apnoea. Thorax. 1997;52(7):648-53.

38. Hack M, Davies RJ, Mullins R, Choi SJ, Ramdassingh-Dow $\mathrm{S}$, Jenkinson C, et al. Randomised prospective parallel trial of therapeutic versus subtherapeutic nasal continuous positive airway pressure on simulated steering performance in patients with obstructive sleep apnoea. Thorax. 2000;55(3):224-31.

39. Mazza S, Pépin JL, Naëgelé B, Rauch E, Deschaux C, Ficheux $\mathrm{P}$, et al. Driving ability in sleep apnoea patients before and after CPAP treatment: evaluation on a road safety platform. Eur Respir J. 2006;28(5):1020-8.

40. Engleman HM, Kingshott RN, Wraith PK, Mackay TW, Deary 1J, Douglas NJ. Randomized placebo-controlled crossover trial of continuous positive airway pressure for mild sleep Apnea/Hypopnea syndrome. Am J Respir Crit Care Med. 1999;159(2):461-7.

41. Engleman HM, Martin SE, Deary 1J, Douglas NJ. Effect of continuous positive airway pressure treatment on daytime function in sleep apnoea/hypopnoea syndrome. Lancet. 1994;343(8897):572-5.

42. Ayalon L, Peterson S. Functional central nervous system imaging in the investigation of obstructive sleep apnea. Curr Opin Pulm Med. 2007;13(6):479-83.

\section{Sobre os autores}

\section{Pedro Felipe Carvalhedo de Bruin}

Professor Associado. Departamento de Medicina Clínica, Faculdade de Medicina, Universidade Federal do Ceará, Fortaleza (CE) Brasil.

\section{Mauricio da Cunha Bagnato}

Responsável Médico. Serviço de Medicina do Sono, Hospital Sírio Libanês, São Paulo (SP) Brasil.

E-mail para contato: pfelipe@superig.com.br (P. Bruin) 